\title{
Reduced Gja5 expression in arterial endothelial cells impairs arteriogenesis during acute ischemic cardiovascular disease
}

\author{
XIANG-JUN LU ${ }^{1}$ and HAI-TAO WANG ${ }^{2}$ \\ ${ }^{1}$ Radiological and ${ }^{2}$ Cardiothoracic Departments, \\ Zhejiang Provincial People's Hospital, Hangzhou, Zhejiang 310014, P.R. China
}

Received January 3, 2015; Accepted February 1, 2017

DOI: $10.3892 /$ etm.2017.5068

\begin{abstract}
The aim of the present study was to investigate the functional role of gap junction protein $\alpha 5$ (Gja5) in arterial endothelial cells in the arteriogenesis that occurs during acute ischemic cardiovascular disease. Gja5 knockout mice and the femoral artery occlusion (FAO) model were used in the current study. Perfusions of both hindlimbs were obtained separately prior to FAO, immediately following FAO and 1, 3, 7, 14 and 21 days after FAO using a Laser Doppler Flow Imager. Genetic evidence concerning the gastrocnemicus (GC) muscle was collected by reverse transcription-quantitative polymerase chain reaction. There were significant reductions in the hindlimb perfusion of Gja5-/- mice compared with Gja5+/+ mice 1, 3, 7, 14 and 21 days following FAO. In Gja5+/- and in $\mathrm{Gja} 5+/+$ mice, the expression of $\mathrm{Gja} 5$ in the GC muscle was increased 4-fold in the ischemic hindlimb 3 days following FAO. Levels of Gja5 expression then returned to baseline values 7 days after FAO. The results of the present study demonstrated that arterial Gja5 expression serves a functional role in acute ischemic cardiovascular disease.
\end{abstract}

\section{Introduction}

Cardiovascular disease is the leading cause of mortality globally (1). Arteries are the primary vessels affected in cardiovascular disease, thus studies investigating the mechanisms of arterial growth and repair are very important. Although successful therapies such as percutaneous coronary intervention (2) and coronary artery bypass graft (3) exist to reduce plaque formation and restore blood flow in patients suffering from ischemic cardiovascular disease (1), a significant proportion of patients do not benefit from such treatment options. It is known that patients with coronary heart disease can recruit collateral vessels, thus experiencing an improvement

Correspondence to: Dr Hai-Tao Wang, Cardiothoracic Department, Zhejiang Provincial People's Hospital, 158 Shangtang Road, Hangzhou, Zhejiang 310014, P.R. China

E-mail: haitaowang1@sina.com

Key words: acute ischemic cardiovascular disease, arteriogenesis, gap junction protein $\alpha 5$, mouse in symptoms (1). The postnatal vascular system is critical for maintaining homeostasis and adapts readily to environmental cues, and physiological or pathological conditions (4). Two different responses, angiogenesis and arteriogenesis (5), occur during this adaptation.

Vascular function is regulated by intercellular communication $(6,7)$. In the vessel wall, cell-to-cell communication occurs by extracellular diffusion and convection of humoral factors, or by the intercytoplasmic exchange of small signaling molecules $(<1 \mathrm{kDa})$, metabolites and ions across gap junctions (6). Endothelial gap junctions are channels that allow but strictly regulate communication between endothelial cells, adjacent smooth muscle and circulating blood cells, as well as throughout the endothelial monolayer (6). Gap junction protein $\alpha 5$ (Gja5, also known as Connexin-40) is the constitutive vascular gap junction protein across species and the vascular bed, and serves an important role in coupling between cells in the vascular wall. Gja5, is essential for a number of physiological processes, particularly in the response to changes in the metabolic demands of tissues $(8,9)$. However, the mechanism of the arterial specific regulation of connexins remains unknown $(10,11)$.

It has been hypothesized that arterial Gja5 expression serves a functional role in flow driven arteriogenesis. Therefore, the current study used Gja5 mutant mice and set up a model of femoral artery occlusion (FAO). Subsequently, blood flow was assessed using Laser Doppler blood flow (LDF) imaging. Furthermore, genetic evidence of mice demonstrating the functional importance of $\mathrm{Gja} 5$ in acute ischemic cardiovascular disease was obtained.

\section{Materials and methods}

Experimental animals. To determine the function of Gja5 in mouse arteriogenesis, CX40 enhanced-green fluorescent protein (EGFP) knock in reporter mice and Gja5 mutant mice from the Max Delbrück Center for Molecular Medicine (Berlin, Germany) were used in this study. The present study used 20 reporter mice and $168 \mathrm{Gja} 5$ mutant mice. The reporter mice are CX40 enhanced-green fluorescent protein (EGFP) knock in mice. This mutant mouse has a CX40 promoter, following with an EGFP gene, which means that whenever there is CX40 gene expression (such as in the arterial endothelial cells), there is GFP expression as well. These mutant mice were described 
by Chadjichristos et al (12) and Miquerol et al (13), respectively. A total of 168 experimental mice were divided into three groups: Gja5-/- group (Cx40 knockdown), Gja5+/- group (Cx40 het) and Gja5+/+ group (wild type; wt). Each group contained 56 mice. All experiments involving animals were performed according to institutional and National Institutes of Health guidelines (Using Animals in Intramural Research) (14) and the protocol was approved by the local ethics committee (Zhejiang Provincial People's Hospital, Hangzhou, China).

Femoral artery occlusion (FAO) model. FAO results in flow driven formation of an arterial collateral network, which increases blood flow to the ischemic hindlimb (15). Occlusion of the right femoral artery in 12 week-old mice was performed as previously described (16).

Mice were anesthetized with an intraperitoneal injection of $100 \mathrm{mg} / \mathrm{kg}$ ketamine $(100 \mathrm{mg} / \mathrm{ml}$; Pharmacia; Pfizer, New York, NY, USA) and $10 \mathrm{mg} / \mathrm{kg}$ xylazine $(20 \mathrm{mg} / \mathrm{ml}$; Bayer Vital GmbH, Leverkusen, Germany) and placed in a supine position. The right inguinal area was shaved and disinfected with $70 \%$ ethanol. The femoral artery was then exposed and separated from the vein and nerve. The two ligations required for the FAO were conducted according to Hoefer's method (16). The proximal circumflex femoral artery is very closely connected to the lateral caudal femoral artery. Therefore, the upper ligation was performed proximally to both branches and the second ligation was conducted below both branches. The femoral artery was then split into the saphenous and popliteal artery. The second ligation was placed proximally to this position and the wounds were subsequently closed.

Assessment of blood flow with Laser Doppler Flow (LDF) imaging. For repeated assessment of hindlimb blood flow following FAO, the non-invasive LDF imaging technique was used (15). The Doppler signal is linearly proportional to perfusion of the upper $200-300 \mu \mathrm{m}$ of the skin (17). Tissue perfusion is quantified in regions of interest, defined in the limbs relative to the contralateral, non-ligated side, and the results are presented as color-coded images (18). Laser Doppler Imaging measurements were taken from the feet, as these measurements correlate with other measures of limb perfusion (19). Following anaesthesia with an intraperitoneal injection of $100 \mathrm{mg} / \mathrm{kg}$ ketamine (100 mg/ml; Pharmacia; Pfizer) and $10 \mathrm{mg} / \mathrm{kg}$ xylazine $(20 \mathrm{mg} / \mathrm{ml}$; Bayer Vital $\mathrm{GmbH})$ of animals, perfusions of both hindlimbs were obtained separately prior to FAO, immediately following FAO, and 1, 3, 7, 14 and 21 days after FAO using a scanning Laser Doppler Flow Imager (model LDI2-HR, Moor Instruments, Axminster, UK).

Demonstration for the collaterals in wt mice 7 days post FAO. The collaterals in wt mice 7 days post FAO were demonstrated through using a Leica Fluorescent microscope at a magnification of x7.5 (Leica Microsystems, GmbH, Watzlar, Germany).

In vitro experiment. To measure Gja5 mRNA expression in gastrocnemicus (GC) muscle, 8 mice from each group: Gja5+/+ and Gja5+/- were sacrificed on day 7 after FAO by cervical dissociation. The gastrocnemicus muscles were selected as it is related to the femoral artery (20). Skin and fasciae were removed from the thighs and lower limbs of the ligated and non-ligated sides of the animal. The GC muscle was isolated and excised, and immediately frozen in liquid nitrogen at $-80^{\circ} \mathrm{C}$. Total RNA of the GC muscle was isolated using TRIzol ${ }^{\circledR}$ reagent (Invitrogen; Thermo Fisher Scientific, Inc., Waltham, MA, USA) following the manufacturers protocol. Reverse transcription was performed using the ThermoScript ${ }^{\mathrm{TM}}$ RT-PCR System for First-Strand cDNA Synthesis (catalogue no. 11146024; Thermo Fisher Scientific, Inc.). Subsequently, quantitative polymerase chain reaction (qPCR; Eurogentec, San Diego, CA, USA) was performed using TaqMan probe-based chemistry. Primers were as follows: Forward primer (For Gja5 gene, 5'-3'): CAGCCT GGCTGAACTCTACCA, reverse primer: CTGCCGTGA CTTGCCAAAG and probes: TaqMan probe, CGCTGTCGG ATCTTCTTCCAGCCCAG. Primers were designed using the Primer Express 2.0 software (Applied Biosystems; Thermo Fisher Scientific, Inc.). Real-time PCR amplification reaction was performed on a Sequence Detection System (7900 HT; Applied Biosystems, Foster City, USA) using the Taqman gene expression Master Mix Plus (Eurogentec, Liege, Belgium). The thermal cycling conditions were as follows: $50^{\circ} \mathrm{C}$ for $2 \mathrm{~min}$ and $95^{\circ} \mathrm{C}$ for $10 \mathrm{~min}$ followed by 40 cycles of $95^{\circ} \mathrm{C}$ for $10 \mathrm{sec}$ and $60^{\circ} \mathrm{C}$ for $1 \mathrm{~min}$. PCR was completed according to manufacturer's instructions with 2X TaqMan universal PCR master mix, $900 \mathrm{nM}$ primers and $250 \mathrm{nM}$ probe. Reactions were performed in triplicate. Data were collected and analyzed with the Sequence Detection System 2.3 software (Applied Biosystems; Thermo Fisher Scientific, Inc.). The relative amount of mRNA was calculated following normalization to glyceraldehyde 3-phosphate dehydrogenase (GAPDH; forward primer: GAAGGTGAAGGTCGGAGTC, reverse primer: GAAGATGGTGATGGGATTTC and TaqMan probe: CAA GCTTCCCGTTCTCAGCC). The comparative CT Method $\left(-2^{\Delta \Delta \mathrm{Cq}}\right.$ Method) was used as described in the User Bulletin 2: ABI PRISM 7700 Sequence Detection System.

Statistical analysis. Graphpad Prism 5 software (GraphPad, Inc., La Jolla, CA, USA) was used to analyze the data. Data are presented as mean \pm standard error of the mean. P-values were calculated using paired Student's t-test. $\mathrm{P}<0.05$ was considered to indicate a statistically significant difference.

\section{Results}

Gja5 (Connexin-40) is expressed in mouse arteries. Using CX40EGFP reporter animals, it was demonstrated that the Gja5 was expressed in the hindlimb arteries, assessed using the Leica fluorescent microscope (Fig. 1).

Greater reduction of hindlimb perfusion in Gja5 deficient mice compared with Gja5+/+ mice following FAO. To determine whether $\mathrm{Gja} 5$ serves a functional role in the flow mediated adaptive remodelling of arteries, the hindlimb femoral artery occlusion model was used and Gja5 mutant mice were investigated. To measure hindlimb perfusion following FAO, hindlimb perfusion with LDF imaging was measured at the following time-points: Prior to FAO, immediately following FAO and 1, 3, 7, 14 and 21 days following FAO. LDF imaging demonstrated that there was a greater reduction of hindlimb perfusion in Gja5 deficient 
Table I. Hindlimb perfusion with Laser Doppler Blood Flow imaging were compared among Gja5+/+, Gja5+/- and Gja5-/- mice.

Time of analysis

\begin{tabular}{lccccccc}
\cline { 2 - 7 } Group & Before & Immediately after & Day 1 & Day 3 & Day 7 & Day 14 & Day 21 \\
\hline Gja5-/- & $894.95 \pm 27.46$ & $39.02 \pm 1.66$ & $38.21 \pm 1.54^{\mathrm{b}}$ & $52.33 \pm 3.23^{\mathrm{b}}$ & $112.17 \pm 19.4^{\mathrm{b}}$ & $263.96 \pm 36.2^{\mathrm{a}}$ & $374.53 \pm 61.07^{\mathrm{a}}$ \\
Gja5+/- & $878.9 \pm 33.38$ & $45.85 \pm 4.45$ & $53.9 \pm 9.8$ & $77.22 \pm 16.49$ & $295.93 \pm 12.85$ & $398.7 \pm 40.00$ & $552.68 \pm 25.25$ \\
Gja5+/+ & $932.19 \pm 17.25$ & $37.73 \pm 1.16$ & $56.81 \pm 5.47$ & $85.12 \pm 8.22$ & $240.26 \pm 17.18$ & $396.96 \pm 27.97$ & $500.45 \pm 57.18$
\end{tabular}

${ }^{\mathrm{a}} \mathrm{P}<0.05,{ }^{\mathrm{b}} \mathrm{P}<0.01$ vs. Gja5+/+ mice; Gja5, gap junction protein $\alpha 5$.

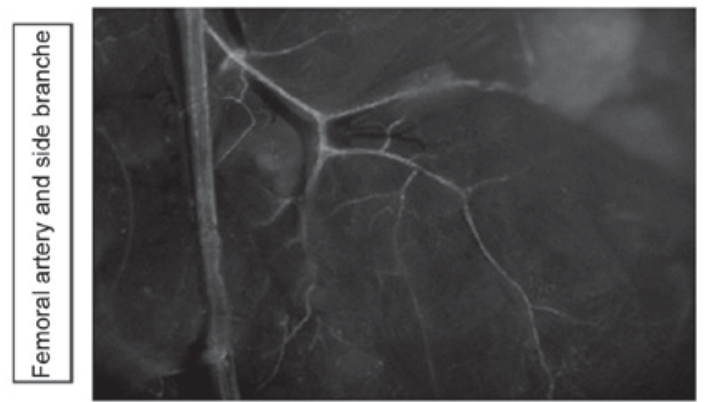

Figure 1. Fluorescence microscopy with a magnification x10 in CX40EGFP reporter animals indicated that gap junction protein $\alpha 5$ was expressed in the hindlimb arteries.

mice compared with Gja5+/+ mice following FAO $(\mathrm{P}<0.05$; Table I).

There was no significant difference in hindlimb perfusion among Gja5+/+, Gja5+/- and Gja5-/- mice prior to FAO and acutely following FAO ( $>>0.05)$; there was a significant reduction in hindlimb perfusion in Gja5-/- mice compared with Gja5+/+ mice 1, 3, 7, 14 and 21 days following FAO $(\mathrm{P}<0.05)$, but there was no difference between Gja5+/- mice and Gja5+/+ mice $(\mathrm{P}>0.05$; Table I).

LDF imaging 3 days following FAO indicated that hindlimb perfusion remained reduced in Gja5-/- mice compared with Gja5+/+ mice ( $\mathrm{P}=0.000187313)$, but there was no difference between Gja5+/- mice and Gja5+/+ mice when compared to Gja5+/+ mice or Gja5+/- mice. LDF imaging 7 days following FAO demonstrated that hindlimb perfusion remained reduced in Gja5-/- mice compared with Gja5+/+ mice ( $\mathrm{P}=0.00036731)$. However, there were no differences between $\mathrm{Gja} 5+/$ - mice and Gja5+/+ mice (Fig. 2).

Fig. 3 demonstrates the collaterals in wt mice 7 days post FAO (7.5x Fluorescence microscopy; Leica Microsystems AG, Heerbrugg, Switzerland) through Leica Fluorescent microscope. No significant difference was observed between Gja5+/- mice and Gja5+/+ mice. The collaterals in wt mice, 7 days post FAO was more than the collaterals in wt mice 3 days post FAO. LDF imaging on day 7 after FAO demonstrated that hindlimb perfusion recovered partly, compared with LDF imaging on day 3.

Expression of Gja5 in the GC muscle following FAO. Using RT-qPCR, expression of Gja5 mRNA was measured in the experimental animals (Fig. 4). Gja5 expression is presented as a ratio from expression in the ischemic hindlimb and the control hindlimb, using the GC muscle of Gja5+/+ and Gja5+/mice ( $\mathrm{n}=8$ animals per group). Levels of $\mathrm{Gja} 5$ expression in Gja5+/- and Gja5+/+ mice were 4-fold higher in the ischemic hindlimb 3 days following FAO. No significant difference was observed in Gja5 expression in gastrocnemius muscle between Gja5+/- mice and Gja5+/+ mice (Fig. 4). Subsequently, levels of Gja5 returned to baseline values at 7 days after FAO.

\section{Discussion}

Arteriogenesis, the growth of collateral vessels, is triggered by fluid shear stress (FSS) that occurs due to arterial occlusion (21-23). It is an important focus of current cardiovascular research as it may provide novel therapeutic opportunities such as percutaneous coronary intervention and coronary artery bypass graft $(24,25)$. Patients with coronary heart disease are able to recruit collateral vessels, thus experiencing an improvement in symptoms. However, the molecular mechanisms responsible for arteriogenesis remain unknown $(26,27)$.

Connexins, a type of gap junction protein, are a family of structurally related transmembrane proteins that assemble in vertebrates to form gap junctions. Gap junctions are formed by a pair of hemichannels called connexions, each contributed by one of two neighbouring cells. Gap junctions serve a multifaceted role in the vasculature and are essential for controlling gene expression, vascular development and vascular function. Gap junction function in the vasculature depends on molecular selectivity or the permeability of different vascular connexin isoforms. It has been determined that the processes of angiogenesis and neurogenesis share common molecules and mediators (28), including electrical coupling that involves gap junction proteins or connexions (11).

To determine the potential role of Gja5 in arteriogenesis, Gja5 mutant mice were investigated using a previously established flow driven arteriogenesis model known as the FAO model (15). Surgical ligation of the femoral artery at a specific site triggers the arteriogenesis of small, pre-existing collateral arteries into functional conduit vessels proximally and ischemic angiogenesis distally (15). The vascular response to hindlimb ischemia can be readily evaluated by laser Doppler-based perfusion measurements and histological quantification of arteriogenesis. The current study demonstrated that Gja5 was expressed in the femoral artery. A Laser Doppler Flow imager was used to measure blood flow and the results indicated significantly reduced perfusion 
A

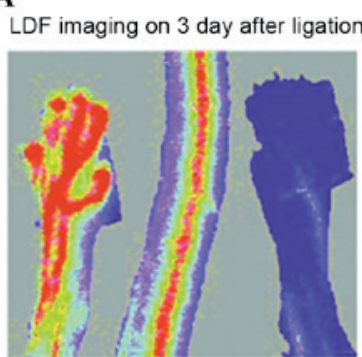

Gja5th mice

B

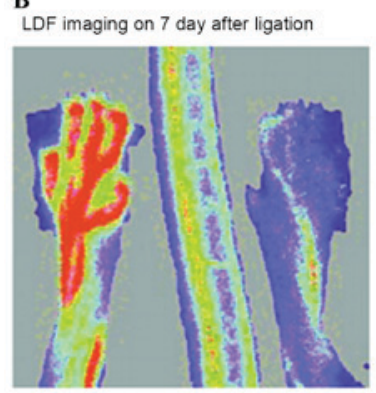

Gja5" mice

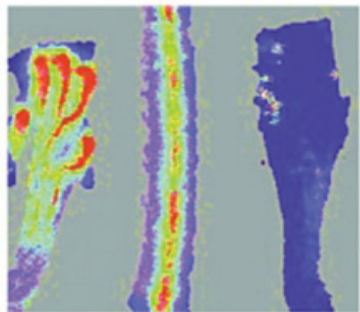

Gja5 mice

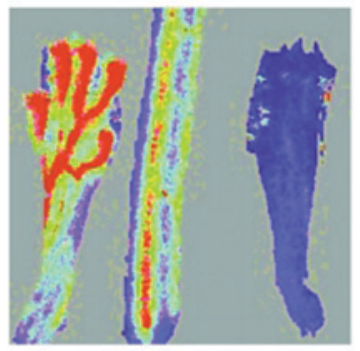

Gja5" mice

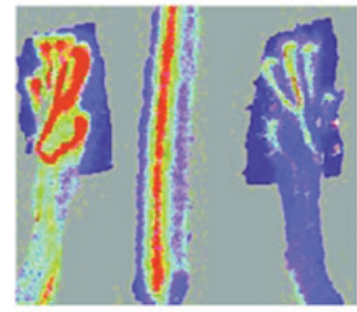

WT mice

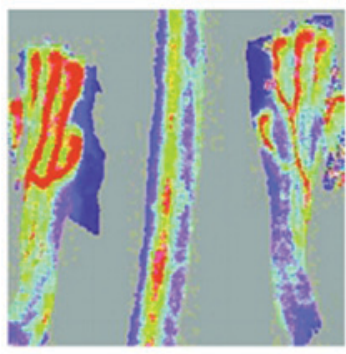

WT mice

Figure 2. (A) LDF measurement showed that hindlimb perfusion remained reduced in Gja5-/- mice compared to Gja5+/+ mice (P=0.000187313) at 3 days after FAO. (B) LDF imaging 7 days after FAO demonstrated that hindlimb perfusion remained reduced in Gja5-/- mice compared with Gja5+/+ mice (P<0.001). LDF, Laser Doppler Blood Flow; FAO, femoral artery occlusion; Gja5, gap junction protein $\alpha 5$.

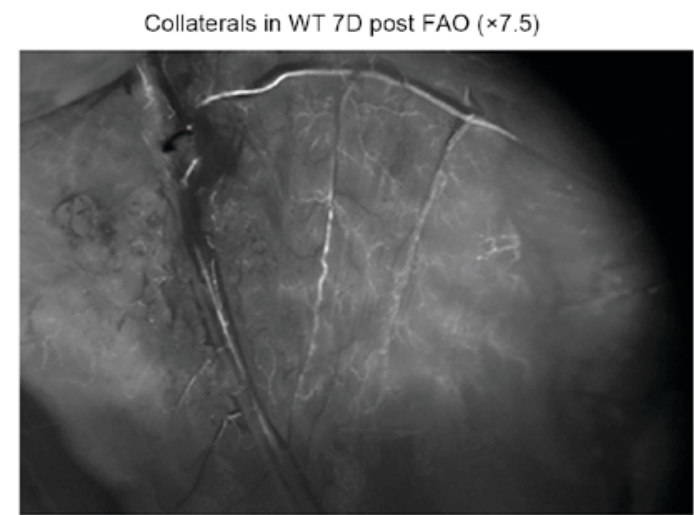

Figure 3. Fluorescence microscopy of the collaterals with a magnification $\mathrm{x} 7.5$ in wt mice 7 days post FAO. No significantly difference was observed between Gja5+/- mice and Gja5+/+ mice, the collaterals in wt mice 7 days post FAO were more than the collaterals in wt mice 3 days post FAO. LDF imaging on day 7 demonstrated that hindlimb perfusion recovered partly, compared with LDF imaging at day 3. wt, wild type; FAO, femoral artery occlusion; Gja5, gap junction protein $\alpha$ 5; LDF, Laser Doppler Flow.

between days 1 and 21 after FAO in Gja5-/- mice compared with Gja5+/+ mice. However, there were no significant differences in perfusion reduction following FAO in Gja5+/mice compared with Gja5+/+ mice. It was observed that FAO induced Gja5 expression in the ischemic hindlimb to a similar extent in Gja5+/- mice and Gja5+/+ mice. At 3 days after occlusion, expression was elevated 4 -fold in the ischemic hindlimb in Gja5+/- and Gja5+/+ mice. However, levels of Gja5 expression returned to baseline values 7 days following occlusion. Notably, Pipp et al (29) demonstrated the importance of FSS in arteriogenesis using a porcine ischemic hindlimb model with high levels of collateral flow and

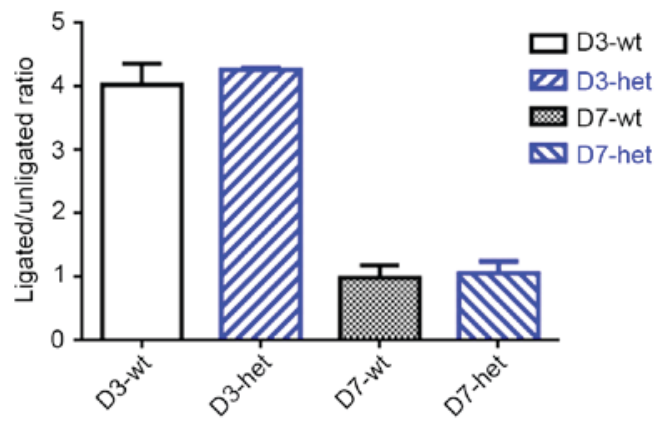

Figure 4. Changes in Gja5 expression in gastrocnemius muscle following FAO. The expression of Gja5 is presented as ratio from expression in the ischemic hindlimb and the control hindlimb, using the gastrocnemius muscle of $\mathrm{Gja} 5+/+$ and $\mathrm{Gja} 5+/-$ mice ( $\mathrm{n}=8$ animals per group). The expression levels of Gja5 in Gja5+/- mice and Gja5+/+ mice were both 4-fold higher in the ischemic hindlimb 3 days after FAO. No significant difference was observed in Gja5 expression in gastrocnemius muscle between $\mathrm{Gja} 5+/$ - mice and Gja5+/+ mice. Then the level of Gja5 expression returned to baseline values 7 days after FAO. FAO, femoral artery occlusion; Gja5, gap junction protein $\alpha 5$; wt, wild type; het, heterozygous.

FSS. Normally, during the later phases of arteriogenesis, FSS decreases as the collateral diameter increases, resulting in the stabilization of FSS. This drop in FSS acts as a signal to arrest proliferation and as a result, prevents further collateral growth prior to optimal adaptation. Therefore, the tendency towards a change in Gja5 expression following FAO is the same as that regarding the change of FSS in arteriogenesis. This suggests that FSS is a dominant morphogenic factor in collateral growth and that Gja5 serves a functional role in arteriogenesis, however further research is necessary to determine the association between Gja5 expression and FSS following FAO. Taken together, the results of the current 
study indicate that Gja5 may function as a positive modulator in arteriogenesis and serve as a general arterial marker.

However, the mechanism underlying arterial specific regulation of connexins remains unknown. Therefore, the present study hypothesizes that numerous genes may be involved in the differential regulation of arteriogenesis, and that a combination analysis of gene expression may help the patient recover from cardiovascular disease.

In conclusion, the current study demonstrated that arterial Gja5 expression serves a functional role in acute ischemic cardiovascular disease and that reduced $\mathrm{Gja} 5$ expression in arterial endothelial cells impairs arteriogenesis.

\section{Acknowledgements}

The present study was supported by the Foundation of Zhejiang Provincial Medical Research (no. 2013ZDA003) and Zhejiang Provincial Qianjiang talent plan D (no. QJD1202017).

\section{References}

1. Hamawy AH, Lee LY, Crystal RG and Rosengart TK: Cardiac angiogenesis and gene therapy: A strategy for myocardial revascularization. Curr Opin Cardiol 14: 515-522, 1999.

2. Pursnani S, Korley F, Gopaul R, Kanade P, Chandra N, Shaw RE and Bangalore S: Percutaneous coronary intervention versus optimal medical therapy in stable coronary artery disease: A systematic review and meta-analysis of randomized clinical trials. Circ Cardiovasc Interv 5: 476-490, 2012.

3. Olearchyk AS: Coronary revascularization: Past, present and future. J Ukr Med Assoc North Am 1: 3-34, 1988.

4. Prior BM, Yang HT and Terjung RL: What makes vessels grow with exercise training? J Appl Physiol (1985) 97: 1119-1128, 2004.

5. Carmeliet P: Mechanisms of angiogenesis and arteriogenesis. Nat Med 6: 389-395, 2000

6. Haefliger JA, Nicod P and Meda P: Contribution of connexins to the function of the vascular wall. Cardiovasc Res 62: 345-356, 2004.

7. Ross R: Cell biology of atherosclerosis. Annu Rev Physiol 57: 791-804, 1995.

8. Lodish HF, Rodriguez RK and Klionsky DJ: Points of view: Lectures: Can't learn with them, can't learn without them. Cell Biol Educ 3: 202-211, 2004.

9. Sohl G and Willecke K: Gap junctions and the connexin protein family. Cardiovasc Res 62: 228-232, 2004.

10. Miquerol L, Meysen S, Mangoni M, Bois P, van Rijen HV, Abran P, Jongsma H, Nargeot J and Gros D: Architectural and functional asymmetry of the His-Purkinje system of the murine heart. Cardiovasc Res 63: 77-86, 2004.

11. Chadjichristos CE, Scheckenbach KE, van Veen TA, Richani Sarieddine MZ, de Wit C, Yang Z, Roth I, Bacchetta M, Viswambharan H, Foglia B, et al: Endothelial-specific deletion of connexin40 promotes atherosclerosis by increasing CD73-dependent leukocyte adhesion. Circulation 121: 123-131, 2010.
12. Chadjichristos CE, Scheckenbach KE, van Veen TA, Richani Sarieddine MZ, de Wit C, Yang Z, Roth I, Bacchetta M, Viswambharan H, Foglia B, et al: Endothelial-specific deletion of connexin 40 promotes atherosclerosis by increasing CD73-dependent leukocyte adhesion. Circulation 121: 123-131, 2010.

13. Miquerol L, Meysen S, Mangoni M, Bois P, van Rijen HV, Abran P, Jongsma H, Nargeot J and Gros D: Architectural and functional asymmetry of the His-Purkinje system of the murine heart. Cardiovasc Res 63: 77-86, 2004.

14. United States Department of Agriculture (USDA): Animal Welfare Act and Animal Welfare Regulations. USDA, Washington, DC, 2013.

15. Hoefer IE, van Royen N, Rectenwald JE, Deindl E, Hua J, Jost M, Grundmann S, Voskuil M, Ozaki CK, Piek JJ and Buschmann IR: Arteriogenesis proceeds via ICAM-1/Mac-1-mediated mechanisms. Circ Res 94: 1179-1185, 2004.

16. Chalothorn D, Zhang H, Clayton JA, Thomas SA and Faber JE: Catecholamines augment collateral vessel growth and angiogenesis in hindlimb ischemia. Am J Physiol Heart Circ Physiol 289: H947-H959, 2005.

17. Jakobsson A and Nilsson GE: Prediction of sampling depth and photon pathlength in laser Doppler flowmetry. Med Biol Eng Comput 31: 301-307, 1993.

18. Chalothorn D, Clayton JA, Zhang H, Pomp D and Faber JE: Collateral density, remodeling, and VEGF-A expression differ widely between mouse strains. Physiol Genomics 30: 179-191, 2007.

19. Helisch A, Wagner S, Khan N, Drinane M, Wolfram S, Heil M, Ziegelhoeffer T, Brandt U, Pearlman JD, Swartz HM and Schaper W: Impact of mouse strain differences in innate hindlimb collateral vasculature. Arterioscler Thromb Vasc Biol 26: 520-526, 2006.

20. Carmen, Schoninger Olaf, Wieser A.A. Ghermanan et al: Anatomy. 2th edition. New Emperor Publishing Ltd., Klagenfurt, pp122-125, 2001 (In German).

21. van Oostrom MC, van Oostrom O, Quax PH, Verhaar MC and Hoefer IE: Insights into mechanisms behind arteriogenesis: What does the future hold? J Leukoc Biol 84: 1379-1391, 2008.

22. Buschmann I and Schaper W: The pathophysiology of the collareral circulation (arteriogenesis). J Pathol 190: 338-342, 2000.

23. Schaper W: Collateral circulation: Past and present. Basic Res Cardiol 104: 5-21, 2009.

24. Heil M and Schaper W: Influence of mechanical, cellular, and molecular factors on collateral artery growth (arteriogenesis). Circ Res 95: 449-458, 2004.

25. Heil M, Eitenmüller I, Schmitz-Rixen T and Schaper W: Arteriogenesis versus angiogenesis: Similarities and differences. J Cell Mol Med 10: 45-55, 2006.

26. Buschmann I and Schaper W: Arteriogenesis versus angiogenesis: Two mechanisms of vessel growth. News Physiol Sci 14: 121-125, 1999.

27. Söhl G and Willecke K: Gap junctions and the connexin protein family. Cardiovasc Res 62: 228-232, 2004.

28. Carmeliet $\mathrm{P}$ and Tessier-Lavigne M: Common mechanisms of nerve and blood vessel wiring. Nature 436: 193-200, 2005.

29. Pipp F, Boehm S, Cai WJ, Adili F, Ziegler B, Karanovic G, Ritter R, Balzer J, Scheler C, Schaper W and Schmitz-Rixen T: Elevated fluid shear stress enhances postocclusive collateral artery growth and gene expression in the pig hindlimb. Arterioscler Thromb Vasc Biol 24: 1664-1668, 2004. 